\title{
Strength Training Volume to Increase Muscle Mass Responsiveness in Older Individuals: Weekly Sets Based Approach
}

\author{
Davi Alves de Santana ${ }^{1,2 *}$, Alex Castro ${ }^{1,3}$ and Cláudia Regina Cavaglieri ${ }^{1}$ \\ ${ }^{1}$ Laboratory of Exercise Physiology, Faculty of Physical Education, University of Campinas, Campinas, Brazil, ${ }^{2}$ Adventist \\ University of Sao Paulo, São Paulo, Brazil, ${ }^{3}$ Nuclear Magnetic Resonance Laboratory, Department of Chemistry, Federal \\ University of São Carlos, São Carlos, Brazil
}

Keywords: resistance training, aging, non-responsiveness, hypertrophy, sets

\section{INTRODUCTION}

Currently, strength training (ST) is widely recommended to promote healthy aging. This reflects efforts made over the past three decades through which the role of ST as a sarcopenia countermeasure, preventing physical disability and other poor outcomes, has become evident. However, in recent years, considerable attention has been directed to the phenomenon of ST response heterogeneity. Different adaptive patterns among individuals submitted to the same intervention have increasingly led scholars to conventionally label non-responders or low responders those who do not respond appropriately, either for lack of meaningful improvements

Edited by:

Robinson Ramírez-Vélez, Public University of Navarre, Spain

Reviewed by:

Eduardo Lusa Cadore, Federal University of Rio Grande do

Sul, Brazil

*Correspondence:

Davi Alves de Santana

das.unicamp@gmail.com

Specialty section:

This article was submitted to

Exercise Physiology,

a section of the journa

Frontiers in Physiology

Received: 16 August 2021 Accepted: 02 September 2021 Published: 30 September 2021

Citation:

Santana DA, Castro $A$ and Cavaglieri CR (2021) Strength Training Volume to Increase Muscle Mass Responsiveness in Older Individuals:

Weekly Sets Based Approach.

Front. Physiol. 12:759677.

doi: 10.3389/fphys.2021.759677 or even for worsening parameters. In the latter case, such individuals are specifically classified generally as adverse responders since they present responses in opposite direction to a threshold theoretically or empirically determined (Bouchard et al., 2012; Hecksteden et al., 2018). Although there is still no consensus on the definition of non-responders or low responders to ST, showing that their study is in its infancy, an interesting review by Atkinson et al. (2019) suggests that the categorization of a given response should be rationalized by the researcher after analyzing which threshold is clinically or practically relevant, changing the notion that minimal detectable change is imperative to determine who respond or not to an intervention.

In this scenario, the phenomenon of non- or low-responsiveness indirectly favored the development of a more restrictive and less important perspective regarding the role of ST. However, reaffirming the ST relevance in aging, Churchward-Venne et al. (2015) have claimed that there are no older non-responders. In that study, it has been shown that all older adults submitted to ST improve at least one analyzed parameter (e.g., functional capacity and muscle strength), defended its widespread application. As suggested by Pickering and Kiely (2019), there are likely no individuals totally unresponsive to training. Nevertheless, the reason why some individuals show less expressive results than others in apparently homogeneous samples remains unclear, especially concerning muscle hypertrophy.

It can be noticed in the study conduct by Churchward-Venne et al. (2015) that more than 35 and $30 \%$ of the older individuals had a maximum lean mass increase of $0.5 \mathrm{~kg}$ after 12 and 24 weeks of ST, respectively. In addition, about $20 \%$ had a decrease in lean mass, regardless of protocol duration. Despite the inherent physiological changes of aging, Ahtiainen et al. (2016) demonstrate that the variability of muscle mass response to ST is not affected by age. Although such changes may indeed lead older individuals to a decreased skeletal muscle tissue sensitivity to anabolic stimuli (Yang et al., 2012), it prohibits putting on account of aging the occurrence of inexpressive morphological adaptations in response 
to training exclusively. Regarding this muscle mass response heterogeneity, while some scholars (Atkinson et al., 2019; Dankel and Loenneke, 2020) recommend cautiousness when claiming its existence, emphasizing that individual differences need to be attested in studies that consider the random error, verified from a matched control group, many others widely recognize it (Hubal et al., 2005; Davidsen et al., 2011; Sparks, 2017; Stec et al., 2017; Camera, 2018; Räntilä et al., 2021). Although more studies need to be conducted to demonstrate the true variability of the response, the body of evidence indicates that such heterogeneity should not be ignored. In this context, it is inevitable to admit that more efforts should be made to clarify the reasons behind the low-responsiveness of some individuals, which, in turn, might assist in planning more effective ST programs.

\section{VOLUME: A KEY POINT?}

The proper design of an ST program involves the management of several variables. Among all, most of them are closely related to intensity and volume. By intensity, it is recognized as the load lifted in a given movement, calculated basically by the percentage of the maximum load lifted only once [e.g., percentage of one-repetition maximum (1RM)]. The training volume, in turn, is traditionally determined by multiplying the number of sets, repetitions, and load. It is noted that the amount of load lifted directly influences the number of repetitions performed (Spiering et al., 2008). Because of the close relationship between repetitions and load, the number of sets performed plays an independent role during the ST progression management and training volume measurement.

Currently, some scholars have considered volume the most effective ST variable to promote morphological changes (Figueiredo et al., 2018). Mattocks et al. (2017) demonstrated that subjects submitted to very high-intensity strength training, simulating $1 \mathrm{RM}$ test sessions, achieved the same strength gains as those who trained at a higher volume. However, only the group that trained with higher volume presented muscle hypertrophy. From a molecular point of view, higher exercise volume positively affects myofibrillar protein synthesis and anabolic signaling and is critical for the degree of p70S6k and S6 phosphorylation following a resistance exercise bout (Burd et al., 2010a,b; Terzis et al., 2010). Additionally, it appears that the type of contraction is not more important than volume to cause molecular changes after an exercise session (Garma et al., 2007). A meta-analysis on the influence of ST on the lean mass of the older individuals found that more significant results were particularly related to protocols where more total sets were performed during the training session (Peterson et al., 2011).

From this evidence, it would be reasonable to expect greater effectiveness of experimental protocols on morphological as higher training volume is applied. Several studies comparing the performance of a single set with multiple sets have demonstrated the superiority of performing multiple sets in both young and older individuals (Kramer, 1997; Radaelli et al., 2014a,b). However, the meta-analysis conducted by Krieger (2010) minimizes the relevance of the volume. In this review, the authors concluded that multiple sets are, in fact, better than a single set but performing four to six sets is not superior to performing two to three sets. While providing valuable information, this body of evidence partially explains why increasing the training volume for more robust physiological responses is not a unanimous strategy (Souza et al., 2018). By the way, it seems that there is a subtle scientific orientation in establishing a minimum dose of exercise by which older people can benefit from ST to the detriment of the search for an optimal dose (Fisher et al., 2017). This is understandable because less time spent on training sessions may increase adherence to the intervention.

These conflicting perspectives raise some unavoidable and related issues. First, does an inadequate training volume explain the unresponsiveness of some older individuals? Consequently, is it possible to establish an optimal volume to maximize hypertrophy in this population? To the best of our knowledge, only one study attempted to answer directly whether training volume is important for the variability of response to ST in older people, despite the large body of studies on this topic (Nunes et al., 2021b). Nunes et al. (2021b) verified that the non-responsiveness condition was not changed after the training volume increase. However, one should be cautious to make conclusions based on this evidence, since it is an analysis of retrospective data in which randomization was not specifically performed to provide a comparative analysis of training volume. On the other hand, Scarpelli et al. (2020) showed that a suboptimal training volume could hamper muscle hypertrophy responses in trained young individuals. This issue is yet to be impulsed by the interesting protocol designed by Montero and Lundby (2017), in which it was observed that all individuals classified as non-responders after 6 weeks of aerobic training responded positively after the additional 2 weeks with higher training volume. Although the intervention of this study has been aerobic training, one can speculate that non-responders to ST also may achieve better adaptations naturally by increasing the training volume.

\section{WEEKLY SETS PER MUSCLE GROUP}

Despite current recommendations on ST for older people (Fragala et al., 2019), which suggests prescribing two to three sets per exercise based on the previously discussed evidence, the optimal training volume to increase muscle mass responsiveness in older individuals is still a scientific challenge. It is proposed here that the findings observed in the literature may be interpreted based, specifically, on the number of weekly sets per muscle group to allow a more comprehensive analysis on this issue, since from this perspective the number of exercise and weekly frequency are already taken into account, avoiding misleading conclusions. It is worth mentioning that these factors usually differ among studies comparing single and multiple sets, which may partially be responsible for obtaining different results. Thus, this approach would contribute to a greater connection among the findings, uniformity in experimental design conception, and practicality in the training 
prescription, emphasizing that few studies analyzed volume from this perspective.

In this regard, an interesting meta-analysis elaborated by Schoenfeld et al. (2017) provides one of the main clues on this matter. They investigated the dose-response relationship between weekly ST volume and muscle mass gains analyzing studies that compared protocols with low and high training volumes. It was noted that ST tends to promote better results with higher doses, further concluding that 10 weekly sets per muscle group or more may be necessary to maximize muscle hypertrophy. Even though this review was not limited to the older individuals, this is consistent with the review proposed by Peterson et al. (2011), although they did not specifically address the number of weekly sets per muscle group. It can be inferred that one of the main strengths of the analysis by Schoenfeld et al. (2017) was to indicate that there may be a training volume threshold to be reached. Importantly, this finding demands a more critical appraisal of current literature since some clinical trials claiming to apply a high training volume may be less than the supposedly required.

While a reduced number of weekly sets may be insufficient to promote more significant hypertrophy, excessive sets may produce prolonged muscle damage and consequently, decrease adaptive response in older people chronically (Hamada et al., 2005; Ferri et al., 2006; Fell and Williams, 2008; Sorensen et al., 2018). Yet, the dose-response of volume on muscle damage in older individuals has to be determined. In this regard, it was observed that 15 weekly sets per muscle group causes more ultrastructure muscle damage in older than young women after a 9-week intervention (Roth et al., 2000) but not in older men when compared to young (Roth et al., 1999). Although one may argue that the high intensity proposed in these studies may have influenced the results, it has been shown that high and lowintensity training with equal volume produces the same level of muscle damage in young people (Paschalis et al., 2005). It is reasonable to suppose that some older people, especially women, do not respond adequately to training due to overtraining, which in turn makes the previous results (Krieger, 2010) understandable since increasing training volume ( 2 to 3 vs. 4 to 6 sets a week) does not necessarily improve response. Therefore, it appears that there is an upper threshold for ST volume in older individuals still have to be confirmed. This observation may be particularly relevant in designing training programs for frail individuals and others with chronic diseases since higher training doses could be more harmful.

In this scenario, training weekly frequency should not be neglected. Dankel et al. (2017) have hypothesized that higher training frequencies may induce a higher hypertrophic response by promoting optimal successive increase in protein synthesis rate. Based on the evidence that there is a plateau in protein synthesis response to increased training volume (Kumar et al., 2012; Martín-Hernández et al., 2013), there would be no advantage in performing a high training volume in a single session. However, it is not possible to make inferences in this regard from the current evidence. A meta-analysis conducted by Schoenfeld et al. (2019) concluded that the ST frequency does not meaningfully impact muscle hypertrophy when the volume is equated. Concerning older individuals, recently Pina et al. (2019) found no difference in lean mass response between individuals who trained two or three times a week after a 12-week setsequated ST. On the other hand, Zaroni et al. (2019) found that high-frequency training (five vs. one time per week) may confer a superior hypertrophic response in young individuals. Regarding the comparison of protocols, Nunes et al. (2021a) argued that weekly set-equated do not necessarily mean volume-equated when there are marked differences in the weekly frequency $(\geq 3)$ since a reduction in the number of repetitions performed in groups with low frequency is observed due to fatigue experienced during the sessions. Nevertheless, some studies that compared protocols with markedly different frequencies with weekly setsequated did not find differences in the increase in muscle hypertrophy (Gomes et al., 2019; Saric et al., 2019).

We recognize that further research needs to be carried with the older population in this sense, as previously suggested (Dankel et al., 2017). Meanwhile, the number of weekly sets needed to enhance muscle hypertrophy in low-responsive older individuals could be achieved by following the ST current position, which recommends a frequency of two to three times per week, comprising one to two exercise for each muscle group per session (Fragala et al., 2019), depending, however, on sets number per exercise, in which it must be individually adjusted during the exercise program.

\section{WHAT IS NEXT?}

To provide more specific recommendations regarding the proper range of weekly sets per muscle group to increase responsiveness to $\mathrm{ST}$ in older people, it is first necessary that the scientific community does not ignore the heterogeneity of the exercise response, reflected, often, by neglecting individual responses in clinical trials. This simple but significant perspective may contribute greatly to the characterization of the nonresponsiveness phenomenon and its prevalence, especially with respect to muscle mass. In particular, there should be an effort to define the minimal clinically important difference regarding muscle hypertrophy in older individuals to support responsiveness categorization, since such difference hardly coincides with the minimum detectable change (Atkinson et al., 2019). Thus, clinical trials assessing relevant clinical outcomes together with hypertrophy response at different times of intervention are required. Second, as a few reviews addressing the dose-response effects of training volume in the older individuals were inconclusive (Steib et al., 2010; Nicola and Catherine, 2011; Silva et al., 2014; Borde et al., 2015) or did not assess morphological adaptations (Rhea et al., 2003), systematic reviews and meta-analysis on the dose-response relationship between weekly sets per muscle group, rather than exercise general sets or weekly frequency, and morphological adaptations in this population are encouraged. It is worth mentioning, none of the reviews cited above considered or measured individual responses. Third, dose-response should be investigated through within-subject experimental protocols and long-term interventions. On the latter, if, on the one hand, 
the duration of the intervention has been considered one of the most relevant ST variables for elderly individuals (Nicola and Catherine, 2011; Radaelli et al., 2014b), on the other hand, prolonged interventions could minimize the influence of the training volume on the hypertrophic response (Da Silva et al., 2018; Teodoro et al., 2019). In future investigations, it is imperative to separate the true interindividual response variance from other sources of variance, since the individual observed changes are the sum of the change caused by the training program, plus the change that would have occurred in the absence of intervention (in the control group), plus measurement error and day-to-day biological variability (Ross et al., 2019). We also emphasize controlling confounders such as concerning the dietary pattern including energy balance and protein intake, given their potential role on muscle mass maintenance (Houston et al., 2008; Hanach et al., 2019; Landi et al., 2019). In addition, possible differences between lower and upper-body muscle group's responses to training should be considered, as there is evidence that high-volume training seems to be more efficient for lower-body muscles (Radaelli et al., 2014b). Lastly, alternative assessments are necessary to understand the variability of the response and assist in planning new studies. In this sense, omic sciences (e.g., genomics, proteomics, and metabolomics) emerge as the next frontier to be explored in this field of knowledge (Tanaka et al., 2016; Picca et al., 2019). From them, diverse dimensions of the organism (e.g., metabolic and genetic) are analyzed simultaneously and, when associated with training responses, can create network maps that help to explain the complexity of morphological adaptations to ST.

\section{CONSIDERATIONS}

Strength training is an important strategy against poor outcomes during aging. In order to enable that older individuals plenty achieve positive adaptations, training variables need to be

\section{REFERENCES}

Ahtiainen, J. P., Walker, S., Peltonen, H., Holviala, J., Sillanpää, E., Karavirta, L., et al. (2016). Heterogeneity in resistance training-induced muscle strength and mass responses in men and women of different ages. Age 38:10. doi: 10.1007/s11357-015-9870-1

Atkinson, G., Williamson, P., and Batterham, A. M. (2019). Issues in the determination of 'responders' and 'non-responders' in physiological research. Exp. Physiol. 104, 1215-1225. doi: 10.1113/EP087712

Borde, R., Hortobágyi, T., and Granacher, U. (2015). Dose-Response relationships of resistance training in healthy old adults: a systematic review and metaanalysis. Sports Med., 45, 1693-1720. doi: 10.1007/s40279-015-0385-9

Bouchard, C., Blair, S. N., Church, T. S., Earnest, C. P., Hagberg, J. M., Häkkinen, K., et al. (2012). Adverse metabolic response to regular exercise: is it a rare or common occurrence? PLoS ONE 7:e37887. doi: 10.1371/journal.pone.0037887

Burd, N. A., Holwerda, A. M., Selby, K. C., West, D. W., Staples, A. W., Cain, N. E., et al. (2010a). Resistance exercise volume affects myofibrillar protein synthesis and anabolic signalling molecule phosphorylation in young men. J. Physiol. 588, 3119-3130. doi: 10.1113/jphysiol.2010.192856

Burd, N. A., West, D. W., Staples, A. W., Atherton, P. J., Baker, J. M., Moore, D. R., et al. (2010b). Low-load high volume resistance exercise stimulates muscle carefully manipulated. In this context, although it is recognized that the training volume is fundamental for muscle hypertrophy, there is no consensus on the magnitude of its influence. Consequently, the contribution level of the volume training for increasing responsiveness to ST in older individuals is lacking. It is noticeable that establishing such a volume is a challenge to be addressed. While future studies are expected to clarify this issue, it can be hypothesized, based on current evidence, that there should be a more specific range of training volume that older people can respond more adequately. We suggested that such a range could be based on the number of sets per muscle group, comprising at least 10 sets per week. Evidently, this does not mean that older individuals do not respond to other training volumes (e.g., <10), but only that some nonresponders or low-responders may have better morphological adaptations in a specific range. In practical terms, after an initial adaptation period, the training volume can be allocated in this range and adjusted according to individual responses to achieve better results. To reach this number of sets, one must carefully select exercises for each muscle group and weekly frequency. In addition, reporting ST volume based on weekly sets per group muscle in future studies may facilitate literature analysis as it already includes the aforementioned variables.

\section{AUTHOR CONTRIBUTIONS}

DS contributed to the conception of the study and wrote the first draft of the manuscript. AC and CC revised the original manuscript critically for important intellectual content. All authors read and approved the final manuscript.

\section{FUNDING}

AC was supported by the São Paulo Research Foundation (FAPESP, Grant No. 2020/13939-7). protein synthesis more than high-load low volume resistance exercise in young men. PLoS ONE 5:e12033. doi: 10.1371/journal.pone.0012033

Camera, D. M. (2018). Anabolic heterogeneity following resistance training: a role for circadian rhythm? Front. Physiol. 9:569. doi: 10.3389/fphys.2018.00569

Churchward-Venne, T. A., Tieland, M., Verdijk, L. B., Leenders, M., Dirks, M. L., De Groot, L. C., et al. (2015). There are no nonresponders to resistance-type exercise training in older men and women. J. Am. Med. Dir. Assoc. 16, 400-411. doi: 10.1016/j.jamda.2015.01.071

Da Silva, L. X. N., Teodoro, J. L., Menger, E., Lopez, P., Grazioli, R., Farinha, J., et al. (2018). Repetitions to failure versus not to failure during concurrent training in healthy elderly men: a randomized clinical trial. Exp. Gerontol. 108, 18-27. doi: 10.1016/j.exger.2018.03.017

Dankel, S. J., and Loenneke, J. P. (2020). A method to stop analyzing random error and start analyzing differential responders to exercise. Sports Med. 50, 231-238. doi: 10.1007/s40279-019-01147-0

Dankel, S. J., Mattocks, K. T., Jessee, M. B., Buckner, S. L., Mouser, J. G., Counts, B. R., et al. (2017). Frequency: the overlooked resistance training variable for inducing muscle hypertrophy? Sports Med. 47, 799-805. doi: 10.1007/s40279-016-0640-8

Davidsen, P. K., Gallagher, I. J., Hartman, J. W., Tarnopolsky, M. A., Dela, F., Helge, J. W., et al. (2011). High responders to resistance exercise training demonstrate 
differential regulation of skeletal muscle microRNA expression. J. Appl. Physiol. 110, 309-317. doi: 10.1152/japplphysiol.00901.2010

Fell, J., and Williams, D. (2008). The effect of aging on skeletal-muscle recovery from exercise: possible implications for aging athletes. J. Aging Phys. Act. 16, 97-115. doi: 10.1123/japa.16.1.97

Ferri, A., Narici, M., Grassi, B., and Pousson, M. (2006). Neuromuscular recovery after a strength training session in elderly people. Eur. J. Appl. Physiol. 97, 272-279. doi: 10.1007/s00421-006-0168-y

Figueiredo, V. C., de Salles, B. F., and Trajano, G. S. (2018). Volume for muscle hypertrophy and health outcomes: the most effective variable in resistance training. Sports Med. 48, 499-505. doi: 10.1007/s40279-017-0793-0

Fisher, J. P., Steele, J., Gentil, P., Giessing, J., and Westcott, W. L. (2017). A minimal dose approach to resistance training for the older adult; the prophylactic for aging. Exp. Gerontol., 99, 80-86. doi: 10.1016/j.exger.2017.09.012

Fragala, M. S., Cadore, E. L., Dorgo, S., Izquierdo, M., Kraemer, W. J., Peterson, M. D., et al. (2019). Resistance training for older adults: position statement from the national strength and conditioning association. J. Strength Cond. Res. 33, 2019-2052. doi: 10.1519/JSC.0000000000003230

Garma, T., Kobayashi, C., Haddad, F., Adams, G. R., Bodell, P. W., and Baldwin, K. M. (2007). Similar acute molecular responses to equivalent volumes of isometric, lengthening, or shortening mode resistance exercise. J. Appl. Physiol. 102, 135-143. doi: 10.1152/japplphysiol.00776.2006

Gomes, G. K., Franco, C. M., Nunes, P. R. P., and Orsatti, F. L. (2019). High-frequency resistance training is not more effective than low-frequency resistance training in increasing muscle mass and strength in well-trained men. J. Strength Cond. Res. 33, S130-S139. doi: 10.1519/JSC.0000000000002559

Hamada, K., Vannier, E., Sacheck, J. M., Witsell, A. L., and Roubenoff, R. (2005). Senescence of human skeletal muscle impairs the local inflammatory cytokine response to acute eccentric exercise. FASEB J. 9, 264-266. doi: 10.1096/fj.03-1286fje

Hanach, N. I., McCullough, F., and Avery, A. (2019). The impact of dairy protein intake on muscle mass, muscle strength, and physical performance in middleaged to older adults with or without existing sarcopenia: a systematic review and meta-analysis. Adv. Nutr. 10, 59-69. doi: 10.1093/advances/nmy065

Hecksteden, A., Pitsch, W., Rosenberger, F., and Meyer, T. (2018). Repeated testing for the assessment of individual response to exercise training. J. Appl. Physiol. 124, 1567-1579. doi: 10.1152/japplphysiol.00896.2017

Houston, D. K., Nicklas, B. J., Ding, J., Harris, T. B., Tylavsky, F. A., Newman, A. B., et al. (2008). Dietary protein intake is associated with lean mass change in older, community-dwelling adults: the health, aging, and body composition (health ABC) study. Am. J. Clin. Nutr. 87, 150-155. doi: 10.1093/ajcn/87.1.150

Hubal, M. J., Gordish-Dressman, H., Thompson, P. D., Price, T. B., Hoffman, E. P., Angelopoulos, T. J., et al. (2005). Variability in muscle size and strength gain after unilateral resistance training. Med. Sci. Sports Exerc. 37, 964-972. Available online at: https://www.researchgate.net/publication/7794282_Variability_in_ muscle_size_and_strength_gain_after_unilateral_resistance_training

Kramer, J. B. (1997). Effects of single vs multiple sets of weight training: impact of volume, intensity, and variation. J. Strength Cond. Res. 11, 143-147. doi: 10.1519/00124278-199708000-00002

Krieger, J. W. (2010). Single vs. multiple sets of resistance exercise for muscle hypertrophy: a meta-analysis. J. Strength. Cond. Res. 24, 1150-1159. doi: 10.1519/JSC.0b013e3181d4d436

Kumar, V., Atherton, P. J., Selby, A., Rankin, D., Williams, J., Smith, K., et al. (2012). Muscle protein synthetic responses to exercise: effects of age, volume, and intensity. J. Gerontol. A Biol. Sci. Med. Sci. 67, 1170-1177. doi: 10.1093/gerona/gls141

Landi, F., Camprubi-Robles, M., Bear, D. E., Cederholm, T., Malafarina, V., Welch, A. A., et al. (2019). Muscle loss: the new malnutrition challenge in clinical practice. Clin. Nutr., 38, 2113-2120. doi: 10.1016/j.clnu.2018. 11.021

Martín-Hernández, J., Marín, P. J., Menéndez, H., Ferrero, C., Loenneke, J. P., and Herrero, A. J. (2013). Muscular adaptations after two different volumes of blood flow-restricted training. Scand. J. Med. Sci. Sports 23, 114-120. doi: $10.1111 /$ sms. 12036

Mattocks, K. T., Buckner, S. L., Jessee, M. B., Dankel, S. J., Mouser, J. G., and Loenneke, J. P. (2017). Practicing the test produces strength equivalent to higher volume training. Med. Sci. Sports Exerc. 49, 1945-1954. doi: 10.1249/MSS.0000000000001300
Montero, D., and Lundby, C. (2017). Refuting the myth of non-response to exercise training: 'non-responders' do respond to higher dose of training. J. Physiol. 595, 3377-3387. doi: 10.1113/JP273480

Nicola, F., and Catherine, S. (2011). Dose-response relationship of resistance training in older adults: a meta-analysis. Br. J. Sports Med. 45, 233-234. doi: $10.1136 /$ bjsm. 2010.083246

Nunes, J. P., Kassiano, W., Costa, B. D. V., Mayhew, J. L., Ribeiro, A. S., and Cyrino, E. S. (2021a). Equating resistance-training volume between programs focused on muscle hypertrophy. Sports Med. 51, 1171-1178. doi: 10.1007/s40279-021-01449-2

Nunes, J. P., Pina, F. L., Ribeiro, A. S., Cunha, P. M., Kassiano, W., Costa, B. D., et al. (2021b). Responsiveness to muscle mass gain following 12 and 24 weeks of resistance training in older women. Aging Clin. Exp. Res. 33, 1071-1078. doi: 10.1007/s40520-020-01587-z

Paschalis, V., Koutedakis, Y., Jamurtas, A. Z., Mougios, V., and Baltzopoulos, V. (2005). Equal volumes of high and low intensity of eccentric exercise in relation to muscle damage and performance. J. Strength Cond. Res. 19, 184-188. doi: 10.1519/00124278-200502000-00031

Peterson, M. D., Sen, A., and Gordon, P. M. (2011). Influence of resistance exercise on lean body mass in aging adults: a meta-analysis. Med. Sci. Sports Exerc. 43, 249-258. doi: 10.1249/MSS.0b013e3181eb6265

Picca, A., Coelho-Junior, H. J., Cesari, M., Marini, F., Miccheli, A., Gervasoni, J., et al. (2019). The metabolomics side of frailty: toward personalized medicine for the aged. Exp. Gerontol. 126:110692. doi: 10.1016/j.exger.2019. 110692

Pickering, C., and Kiely, J. (2019). Do non-responders to exercise existand if so, what should we do about them? Sports Med. 49, 1-7. doi: 10.1007/s40279-018-01041-1

Pina, F. L. C., Nunes, J. P., Schoenfeld, B. J., Nascimento, M. A., Gerage, A. M., Januário, R. S. B., et al. (2019). Effects of different weekly setsequated resistance training frequencies on muscular strength, muscle mass, and body fat in older women. J. Strength Cond. Res. 34, 2990-2995. doi: 10.1519/JSC.0000000000003130

Radaelli, R., Botton, C. E., Wilhelm, E. N., Bottaro, M., Brown, L. E., Lacerda, F., et al. (2014a). Time course of low- and high-volume strength training on neuromuscular adaptations and muscle quality in older women. Age 36, 881-892. doi: 10.1007/s11357-013-9611-2

Radaelli, R., Wilhelm, E. N., Botton, C. E., Rech, A., Bottaro, M., Brown, L. E., et al. (2014b). Effects of single vs. multiple-set short-term strength training in elderly women. Age 36:9720. doi: 10.1007/s11357-014-9720-6

Räntilä, A., Ahtiainen, J. P., Avela, J., Restuccia, J., Kidgell, D., and Häkkinen, K. (2021). High responders to hypertrophic strength training also tend to lose more muscle mass and strength during detraining than low responders. J. Strength Cond. Res. 35, 1500-1511. doi: 10.1519/JSC.00000000000 04044

Rhea, M. R., Alvar, B. A., Burkett, L. N., and Ball, S. D. (2003). A meta-analysis to determine the dose response for strength development. Med. Sci. Sports Exerc. 35, 456-464. doi: 10.1249/01.MSS.0000053727.63505.D4

Ross, R., Goodpaster, B. H., Koch, L. G., Sarzynski, M. A., Kohrt, W. M., Johannsen, N. M., et al. (2019). Precision exercise medicine: understanding exercise response variability. Br. J. Sports Med. 53, 1141-1153. doi: 10.1136/bjsports-2018-100328

Roth, S. M., Martel, G. F., Ivey, F. M., Lemmer, J. T., Metter, E. J., Hurley, B. F., et al. (2000). High-volume, heavy-resistance strength training and muscle damage in young and older women. J. Appl. Physiol. 88, 1112-1128. doi: 10.1152/jappl.2000.88.3.1112

Roth, S. M., Martel, G. F., Ivey, F. M., Lemmer, J. T., Tracy, B. L., Hurlbut, D. E., et al. (1999). Ultrastructural muscle damage in young vs. older men after highvolume, heavy-resistance strength training. J. Appl. Physiol. 86, 1833-1840. doi: 10.1152/jappl.1999.86.6.1833

Saric, J., Lisica, D., Orlic, I., Grgic, J., Krieger, J. W., Vuk, S., et al. (2019). Resistance training frequencies of 3 and 6 times per week produce similar muscular adaptations in resistance-trained men. J. Strength Cond. Res. 33, S122-S129. doi: 10.1519/JSC.0000000000002909

Scarpelli, M. C., Nóbrega, S. R., Santanielo, N., Alvarez, I. F., Otoboni, G. B., Ugrinowitsch, C., et al. (2020). Muscle hypertrophy response is affected by previous resistance training volume in trained individuals. J. Strength Cond. Res. 27, 1-5. doi: 10.1519/JSC.0000000000003558 
Schoenfeld, B. J., Grgic, J., and Krieger, J. (2019). How many times per week should a muscle be trained to maximize muscle hypertrophy? A systematic review and meta-analysis of studies examining the effects of resistance training frequency. J. Sports Sci. 37, 1286-1295. doi: 10.1080/02640414.2018.1555906

Schoenfeld, B. J., Ogborn, D., and Krieger, J. W. (2017). Dose-response relationship between weekly resistance training volume and increases in muscle mass: a systematic review and meta-analysis. J. Sports Sci. 35, 1073-1082. doi: 10.1080/02640414.2016.1210197

Silva, N. L., Oliveira, R. B., Fleck, S. J., Leon, A. C., and Farinatti, P. (2014). Influence of strength training variables on strength gains in adults over 55 years-old: a meta-analysis of dose-response relationships. J. Sci. Med. Sport 17, 337-344. doi: 10.1016/j.jsams.2013.05.009

Sorensen, J. R., Skousen, C., Holland, A., Williams, K., and Hyldahl, R. D. (2018). Acute extracellular matrix, inflammatory and MAPK response to lengthening contractions in elderly human skeletal muscle. Exp. Gerontol. 106, 28-38. doi: 10.1016/j.exger.2018.02.013

Souza, D. C., Viana, R. B., Coswig, V. S., Fisher, J. P., Steele, J., and Gentil, P. (2018). Comment on: volume for muscle hypertrophy and health outcomes: the most effective variable in resistance training. Sports Med. 48, 1281-1284. doi: 10.1007/s40279-018-0865-9

Sparks, L. M. (2017). Exercise training response heterogeneity: physiological and molecular insights. Diabetologia 60, 2329-2336. doi: 10.1007/s00125-017-4461-6

Spiering, B. A., Kraemer, W. J., Anderson, J. M., Armstrong, L. E., Nindl, B. C., Volek, J. S., et al. (2008). Resistance exercise biology: manipulation of resistance exercise programme variables determines the responses of cellular and molecular signalling pathways. Sports Med. 38, 527-540. doi: 10.2165/00007256-200838070-00001

Stec, M. J., Thalacker-Mercer, A., Mayhew, D. L., Kelly, N. A., Tuggle, S. C., Merritt, E. K., et al. (2017). Randomized, four-arm, dose-response clinical trial to optimize resistance exercise training for older adults with age-related muscle atrophy. Exp. Gerontol. 99, 98-109. doi: 10.1016/j.exger.2017.09.018

Steib, S., Schoene, D., and and, Pfeifer, K. (2010). Dose-response relationship of resistance training in older adults: a meta-analysis. Med. Sci. Sports Exerc. 42, 902-914. doi: 10.1249/MSS.0b013e3181c34465

Tanaka, M., Wang, G., and Pitsiladis, Y. P. (2016). Advancing sports and exercise genomics: moving from hypothesis-driven single study approaches to large multi-omics collaborative science. Physiol. Genomics 48, 173-174. doi: 10.1152/physiolgenomics.00009.2016

Teodoro, J. L., da Silva, L. X. N., Fritsch, C. G., Baroni, B. M., Grazioli, R., Boeno, F. P., et al. (2019). Concurrent training performed with and without repetitions to failure in older men: a randomized clinical trial. Scand. J. Med. Sci. Sports 29, 1141-1152. doi: 10.1111/sms.13451

Terzis, G., Spengos, K., Mascher, H., Georgiadis, G., Manta, P., and Blomstrand, E. (2010). The degree of p70 S6k and S6 phosphorylation in human skeletal muscle in response to resistance exercise depends on the training volume. Eur. J. Appl. Physiol. 110, 835-843. doi: 10.1007/s00421-010-1527-2

Yang, Y., Breen, L., Burd, N. A., Hector, A. J., Churchward-Venne, T. A., Josse, A. R., et al. (2012). Resistance exercise enhances myofibrillar protein synthesis with graded intakes of whey protein in older men. Br. J. Nutr. 108, 1780-1788. doi: 10.1017/S0007114511007422

Zaroni, R. S., Brigatto, F. A., Schoenfeld, B. J., Braz, T. V., Benvenutti, J. C., Germano, M. D., et al. (2019). High resistance-training frequency enhances muscle thickness in resistance-trained men. J. Strength Cond. Res. 33, S140S151. doi: 10.1519/JSC.0000000000002643

Conflict of Interest: The authors declare that the research was conducted in the absence of any commercial or financial relationships that could be construed as a potential conflict of interest.

Publisher's Note: All claims expressed in this article are solely those of the authors and do not necessarily represent those of their affiliated organizations, or those of the publisher, the editors and the reviewers. Any product that may be evaluated in this article, or claim that may be made by its manufacturer, is not guaranteed or endorsed by the publisher.

Copyright (c) 2021 Santana, Castro and Cavaglieri. This is an open-access article distributed under the terms of the Creative Commons Attribution License (CC BY). The use, distribution or reproduction in other forums is permitted, provided the original author(s) and the copyright owner(s) are credited and that the original publication in this journal is cited, in accordance with accepted academic practice. No use, distribution or reproduction is permitted which does not comply with these terms. 